\title{
TECTÓNICA Y MORFOLOGIA DE LA MARGEN CONTINENTAL EN EL SUR DE GUERRERO-OAXACA, MÉXICO, A PARTIR DE DATOS GEOFÍSICOS
}

\author{
José Héctor Sandoval Ochoa*
}

\begin{abstract}
RESUMEN
Una extrapolación del modelo típico de la margen continental activa sugiere un dislocamiento de una porción continental localizada en el sur del Macizo de Guerrero-Oaxaca. El fenómeno consistió en un truncamiento que dislocó hacia el Este a una porción de la margen debido a erosión tectónica. Este trucamiento favoreció a los movimientos verticales del Macizo. En estas condiciones la subsecuente acumulación secuencial de cuñas sedimentarias hechas de turbiditas, pelágicos y hemipelágicos ha tendido a definir un prisma acrecionado a los pies del, así creado, abrupto talud continental interno.
\end{abstract}

Con el modelado geofísico de esta región se intenta explicar la ocurrencia de la fosa de la trinchera debido a electos tensionales en la parte superior de la corteza oceánica en subducción cuando en esta localidad se dobla y se hunde. La evolución de la acrecencia comienza cuando no hay más lugar para la llegada constante de material a un ámbito en estado de esfuerzos compresionales, o bien, en consecuencia a la presencia del abrupto pie del muro continental (límite del truncamiento) en contra de la corteza oceánica en subducción. Así entonces, conforme la corteza oceánica rebasa al eje de la trinchera se desarrolla el fallamiento normal con los planos de falla, en su mayoría, buzando hacia la trinchera. Cuando estos alcanzan al pie del talud, el sistema de fallamiento normal se convierte en uno de fallamiento inverso por debajo del talud interno.

Se concluye que este es un mecanismo que explica el desarrollo de un prisma acrecionado hecho definitivamente de pelágicos, hemipelágicos, turbidíticos, derrumbes de sedimentos y ocasionalmente piezas de basalto de la corteza oceánica en algunas cuñas. Entre esas estructuras en un panorama general, se distingue, a unos pocos kilómetros enfrente del talud interno, un contraste estructural muy evidente, ya que la fosa de la trinchera misma ha sido formada por bloques caídos y hendiduras tensionales. Esta trinchera en algunos lugares está llena de sedimentos y en otras porciones está completamente vacía. Con este análisis se ha podido determinar el marco tectónico resultante para este rasgo regional tan largo. y sin embargo, tan angosto.

*Instituto de Ciencias del Mar y Limnología, UNAM. 


\begin{abstract}
Extrapolation of the typical model for the active continental margin suggests a dislocation of a continental portion located in the south of Guerrero-Oaxaca Masiff. The phenomenon was a truncation that offset crustal piece of the margin toward the east, due lo tectonic erosion. The break up was favorable to vertical movements in the massif. A subsequent sequential emplacement of wedges made up of turbidites and hemipelagic sediments, has tended to define an accretionary prism at the toe of the so created sharp inner slope.
\end{abstract}

The geophysical modeling under this area attempts, to explain the occurrence of the trench-trough due to tensional effects along the subducting oceanic crust when the upper slab bands and plunges. The evolution of accretion starts when there is no space for material input to a compressional ambit, or consequent to existence of a sharp toe the continental wall (truncation boundary) against the subducting oceanic crust. Then, as ocean crust passes the trench axis, normal faulting evolves with the majority of fault planes diping toward the trench. When it reaches the toe of slope, the normal faulting appears to convert to inverse faulting below the inner slope. This is the mechanisms for growth of accretionary prism made up widely of: hemipelagic, turbiditic, slump sediments and occasionally pieces of basalt from the oceanic crust in some wedges. Among these structures in an overall view, a few kilometers in front of the inner slope the contrast is quite apparent since the trench trough itself is made up of down faulting and tensional gaps. This trench in some places is full of sediments and other portions are completely empty. The resulting tectonical setting can be determinated for this narrow but long regional feature. 


\section{GENERALIDADES}

Uno de los capítulos más apasionantes de la Nueva Tectónica Global comprende el estudio de las márgenes de placas tectónicas. En las dos últimas décadas este estudio se llevó a cabo en base de las diferencias entre las regiones de corteza sísmicas. Después de varios modelos propuestos de placas ensambladas, fue necesario dar un movimiento coordinado en el tiempo y en el espacio en la variedad de piezas tectónicas. Las representaciones de las placas móviles tuvieron que cumplir con varios requisitos tales como: un balance de masas en toda clase de interacciones del conjunto de placas móviles, definir la ruta de trayectoria de las placas migrantes en un casquete esférico, concordancia con los puntos pivote cambiantes; así como la influencia variante en el tiempo, del campo geomagnético, registrada en algunas rocas, etcétera. Los conceptos como márgenes de placa "constructivo" y "destructivo" se desarrollaron tras el conocimiento de la distribución de las inversiones magnéticas y asimismo los términos de regiones sísmica o activa y asísmica, se acuñaron de tal modo que los conceptos como márgenes "inactiva" y "pasiva" o "inactiva" han sido aceptados ampliamente para definir los bordes de placas. De manera semejante debido al contacto lateral entre placas en movimiento, los términos como "convergente", "conservativo" y "divergente" se han estado aplicando a las márgenes de placas tectónicas. El estudio de las márgenes de placas circumpacíficas en su "tipo activo, destructivo o convergente" ha recibido especial interés, por ser ahí donde la transferencia de masa tiene lugar o bien, que ésta, aparentemente, tiende a desaparecer.

Todo alrededor, por debajo del Océano Pacífico, las placas y márgenes han definido tendencias de estructuras longitudinales características. Estos rasgos regionales se han identificado a partir de evidencias geofísicas y geológicas a lo largo de alineamientos de varios miles de kilómetros de longitud. Estos lineamientos han sido repartidos dentro de áreas específicas denominadas segmentos. con varios cientos kilómetros a lo largo de la tendencia del sistema de arco, para facilitar su estudio; ahí, tales segmentos están delimitados por varios rasgos morfológicos transversales distintivos (Stoiber y Carr, 1973 Carr et. al. 1974), y otras discontinuidades estructurales.

Uno de esos segmentos, entre otros, se localiza en la margen continental del sur de México, exactamente bajo la zona limítrofe de los estados de Guerrero y Oaxaca (Figura 1), la zona de transición océano-continente (geología continental y marina), la Trinchera Mesoamericana (Stoiber y Carr, op. cit. ) y la Placa de Cocos (Truchan et. al., 1974, Handschumacher, 1976). Todos esos rasgos Carr, op. cit. geográficos y morfológicos asociados en el segmento han sido investigados con los datos geofísicos del Marine Science Institute, The University of Texas at Galveston, (UT-MSI) 1977-1982; en un levantamiento integral que incluye Perfilaje de Reflexión Multicanal continua, Refracción, Magnetometría y Muestreo del fondo 


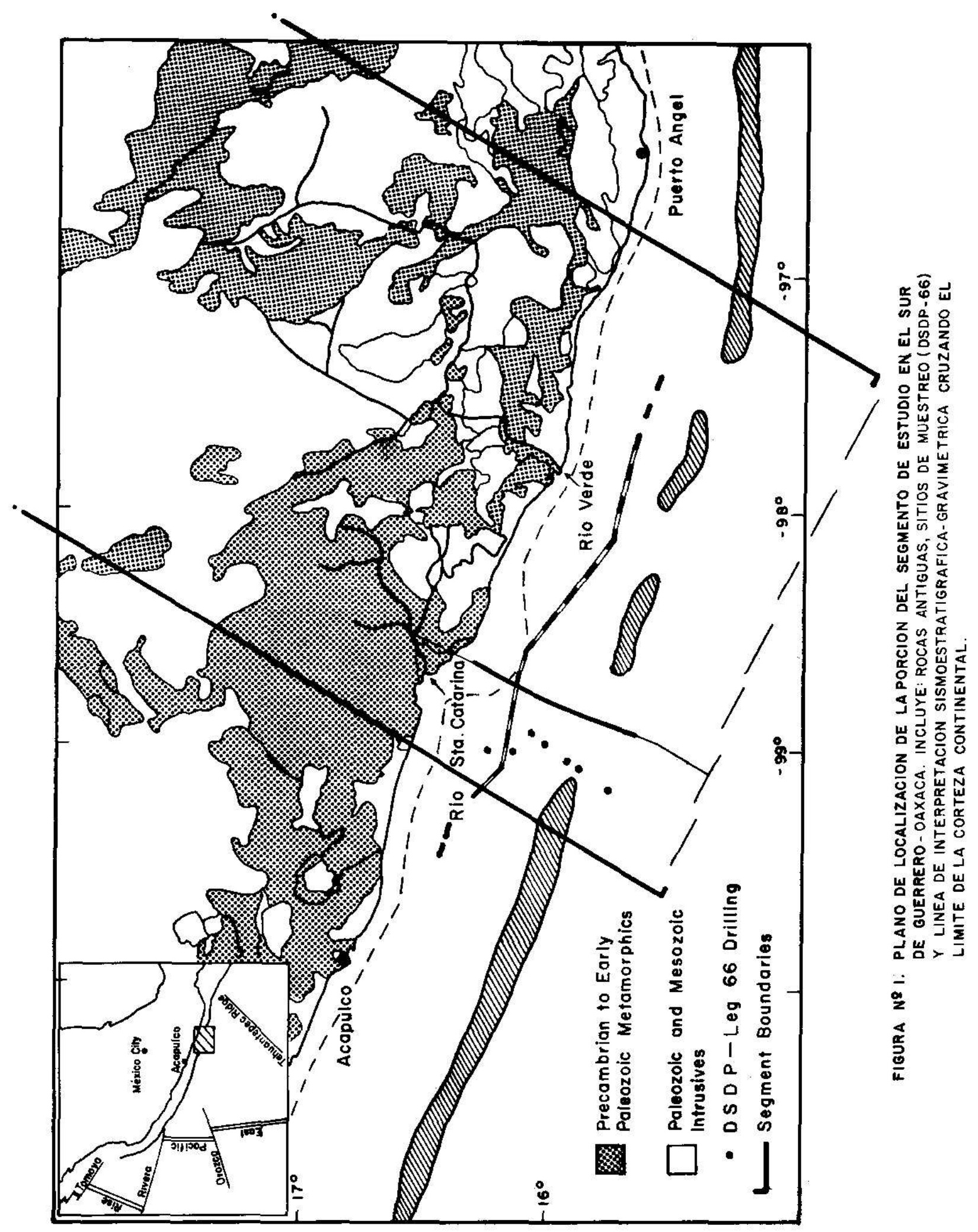


oceánico, donde el autor participó; y asimismo con datos del Proyecto de Perforación de mar profundo Deep Sea Drilling Project, Leg 66, (DSDP).

En este trabajo se maneja con algún detalle la región marginal, donde una versión modificada del modelo de talud continental con trinchera, de Seely-Vall-Walton (1974) se ha aplicado. Con datos gravimétricos se ha creado un modelo nuevo de trabajo, geométrico y teórico, Como resultado obligado de la información sismoestratigráfica y de perforación (Shipley, et.. al. 1980, Shipley, 1981 Watkins, 1981). Este modelo como herramienta de una hipótesis de trabajo incluye una terminación continental abrupta la cual sugiere la dislocación de una porción continental del extremo sur del macizo Guerrero-Oaxaca (Shipley et. al., 1980; Karlg et. al., 1978; Sandoval-Ochoa, 1985). Como resultado de un movimiento sinistral de "rift" de una pieza de la corteza continental en el Terciario (Mellalt, Dinkelman, 1972; Aguayo y Marín, 1987). (Figura 2)

Aparentemente este rompimiento cortical sucedió durante el Eoceno tardío al principio de la época Miocénica y fue favorable a movimientos verticales en el macizo continental de Guerrero-Oaxaca. En consecuencia estos movimientos epeirogénicos fueron previos e independientes a la existencia de un protoprisma cuadrangular rocoso. Acto seguido, los terrenos acrecionarios se desarrollaron en sentidos vertical y hacia el mar para el Mioceno tardío, los cuales continúan hasta épocas Holocénicas
(Shipley et. al., 1980; Watkins et. al., 1981), la expresión de estos terrenos se discute con el modelo aquí propuesto. El prisma acrecionario ha quedado constituido por una acumulación secuencial con carácter inverso de cuñas sedimentarias de depósitos turbidíticos en su mayoría y pelágicos-hemipelágicos en menor proporción.

A partir de los datos de geología superficial de las áreas continental y del mar se han reportado algunos depósitos del Triásico al Paleógeno (Shipley et. al., 1980; De Cserna, 1976; Nell y Moore, 1981) los cuales han sugerido que las discontinuidades laterales de los paquetes estratigráficos se debieron al truncamiento (Karig et. al., 1978) debido, presumiblemente a una gran reorganización de placas, hace no menos 22 Ma (Watkins, et. al., 1981) De acuerdo con las ideas de Karig (1974) y Karig et. al., (1978) sobre los procesos de un "rift" de la margen continental a lo largo de la tendencia del sistema de arco y asimismo, de acuerdo con Watkins et. al., (1981) con la mencionada reorganización; durante la época de 22 a 10 Ma la subducción de carácter oblicuo produjo en consecuencia, una etapa de erosión tectónica intermitente. Finalmente, a partir de $10 \mathrm{Ma}$ hasta el día de hoy, se ha venido desarrollando progresivamente un creciente prisma acrecionario. Durante esta época del Mio-Plioceno al Reciente también evolucionaron las rocas volcánicas de la Faja Volcánica Mexicana (Verma, 1985) asociada a la Trinchera Mesoamericana. 


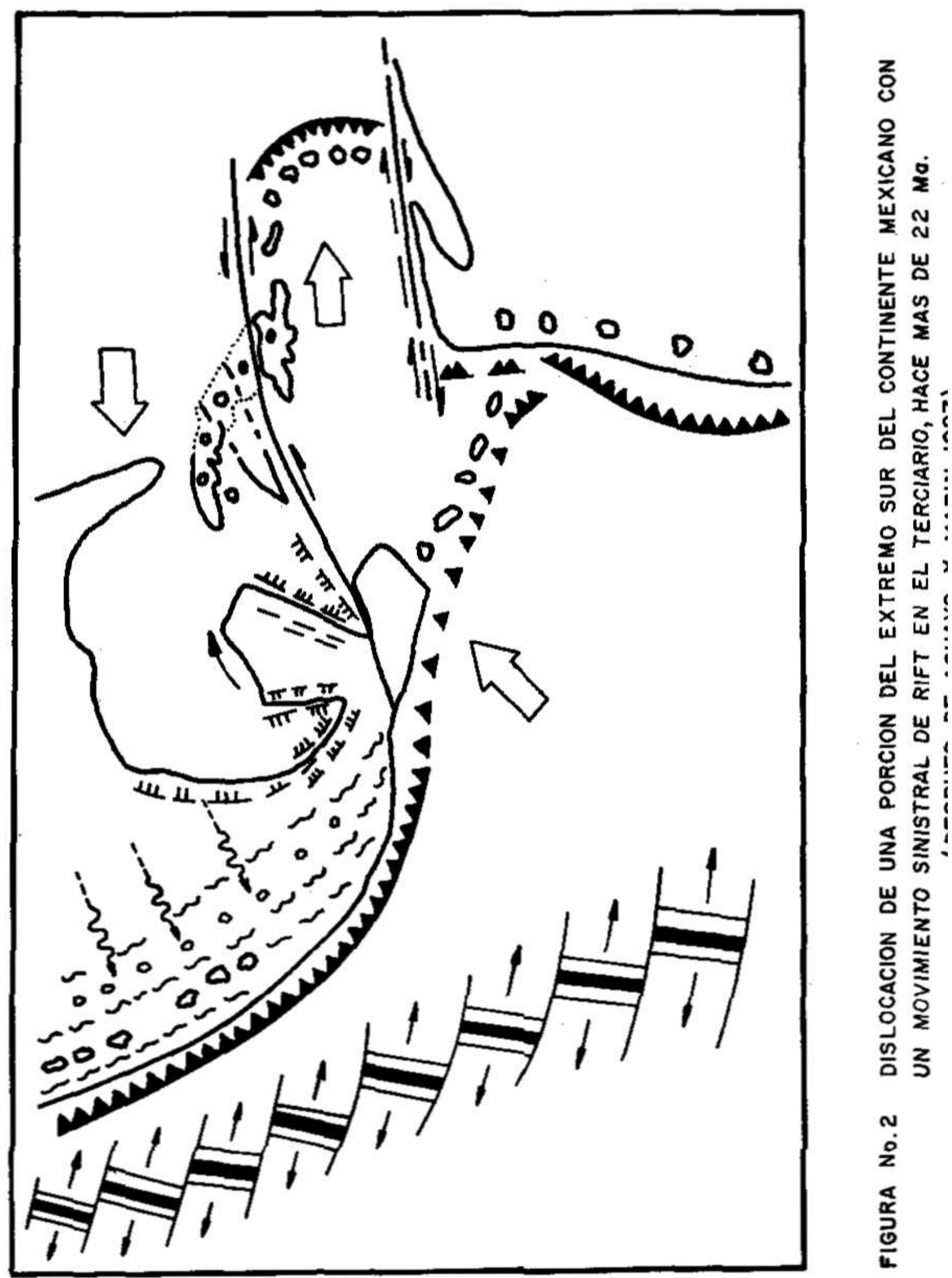




\section{EL MODELO GRAVIMETRICO PARA UNA HIPOTESIS DE TRABAJO.}

Para el prisma acrecional las evaluaciones del balance de masas, en condiciones como las descritas por Watkins et. al., (1981), plantean una hipótesis con una extensión hacia el continente de un modelo de prisma con su cara buzante hacia el continente con 45 NNE. Esta interpretación, no obstante de ser la primera de la zona de transición, proporciona una buena representación al ser comparada con la pared inclinada del continente como una margen abrupta, creada como se ha descrito. De acuerdo con esto, se esperaría que tal plano en el límite continental abrupto del macizo truncado, tiene un echado entre 45 y 61 según su modelo hipotético. Teóricamente, si aceptamos que cualquier losa frágil y tendida, sujeta esfuerzos horizontales de compresión (Fig. 3) rompe en un ángulo entre 45 y 90 con respecto a un plano horizontal; sin embargo, debido al carácter oblicuo de algún empuje por la subducción, el plano de ruptura tenderá a ser más empinado y casi vertical.

En este trabajo, con el objeto de tener un modelo poligonal en dos dimensiones del prisma acrecionado y computarizado, se utiliza la línea OM-6N, (fig. 4), a partir de un arreglo interpretativo de facies y posiciones en un marco geométrico posterior a un análisis sismo estratigráfico. La información del DSDP Leg-66 en vecindad de la línea se integró para restringir el modelado. La computación digital de la anomalía gravimétrica fue llevada a cabo con el algoritmo de
Talwani-2D para gravedad en una versión modificada. Después de pocos intentos, la anomalía calculada se superpuso a la anomalía de gravedad de aire libre dada a UT-MSI por OSU (Couch y Woodcock, 1981), (Fig. 5). Las anomalías se evaluaron en dirección transversal a la tendencia del campo gravífico cortando, asimismo, a los ejes estructurales de la margen continental. Se tuvo una superposición de curvas aceptable para la interpretación con errores tolerables bajo 9 miligales (Flg. 6), incluso siendo válido el modelo y digno para un análisis isostático, y así, cualitativamente comparar con otras áreas, (Watkins et. al., 1981; Garland, 1970; Wollard y Monges, 1956).

\section{DISCUSIÓN}

Típicamente, como es de esperarse, el extremo sur de la línea de análisis (OM-6N) cruza a la anomalía mínima de aire libre negativa la cual se localiza a lo largo de la Trinchera sin ningún cambio sensible a lo largo de su eje dentro del segmento y regiones aledañas. En el otro extremo, en su prolongación hacia el Norte, aún en la porción continental se encuentra un alto gravimétrico que llega hasta +60 mgal, justamente encima del así llamado Banco Tártaro (Tartar Shoal), un alto topográfico reportado previamente por Fisher, 1961 (en: Shipley, 1971; Couch y aqul. Woodcock. 1981), el cual ahora se interpreta como un alto estructural. (Figura No. 5)

Cerca de la porción sur del segmento estudiado se encuentra qué, de la misma manera que la 


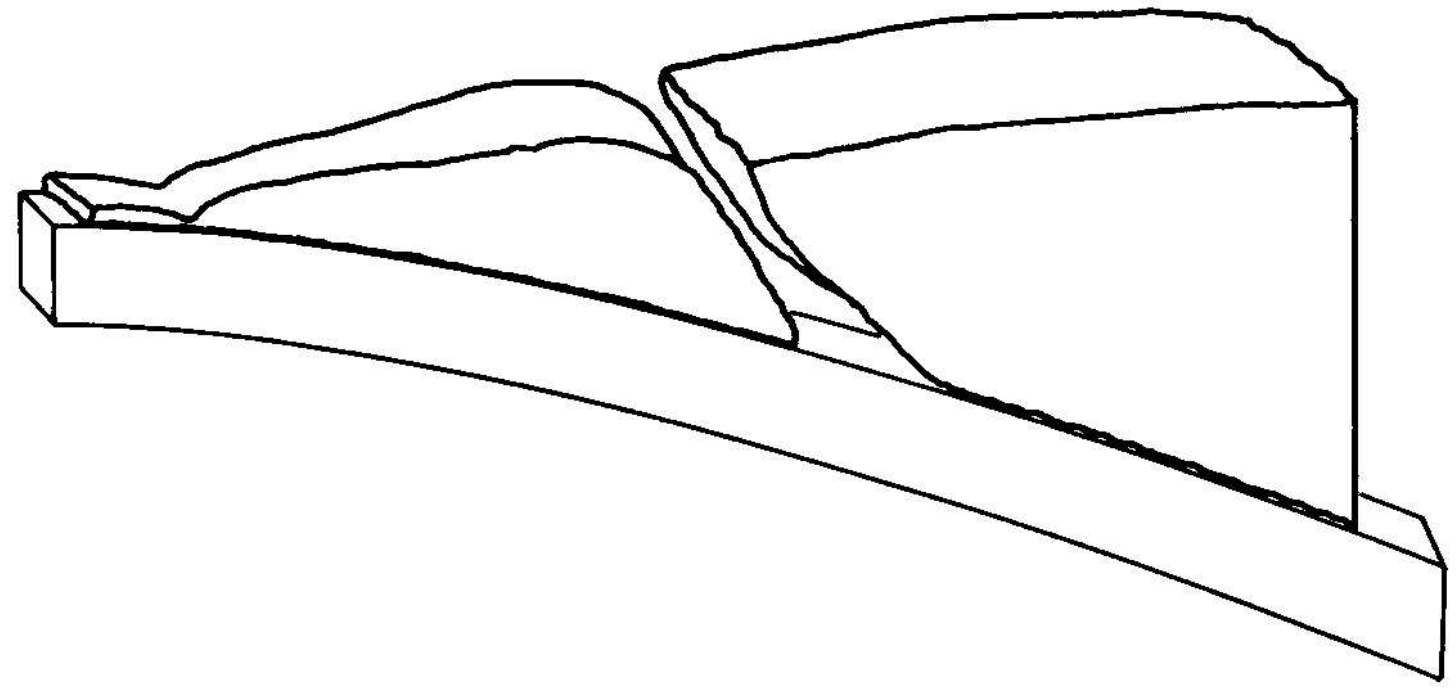

F16. No. 3

-) MOdelo hipotetico del PRISMA ACREcionario de LA TRINCHERA MESOAMERICAMA EN LA MARGEN CONTINENTAL. DEL SUR DE GUERRERO-OAXACA, MEXICO.

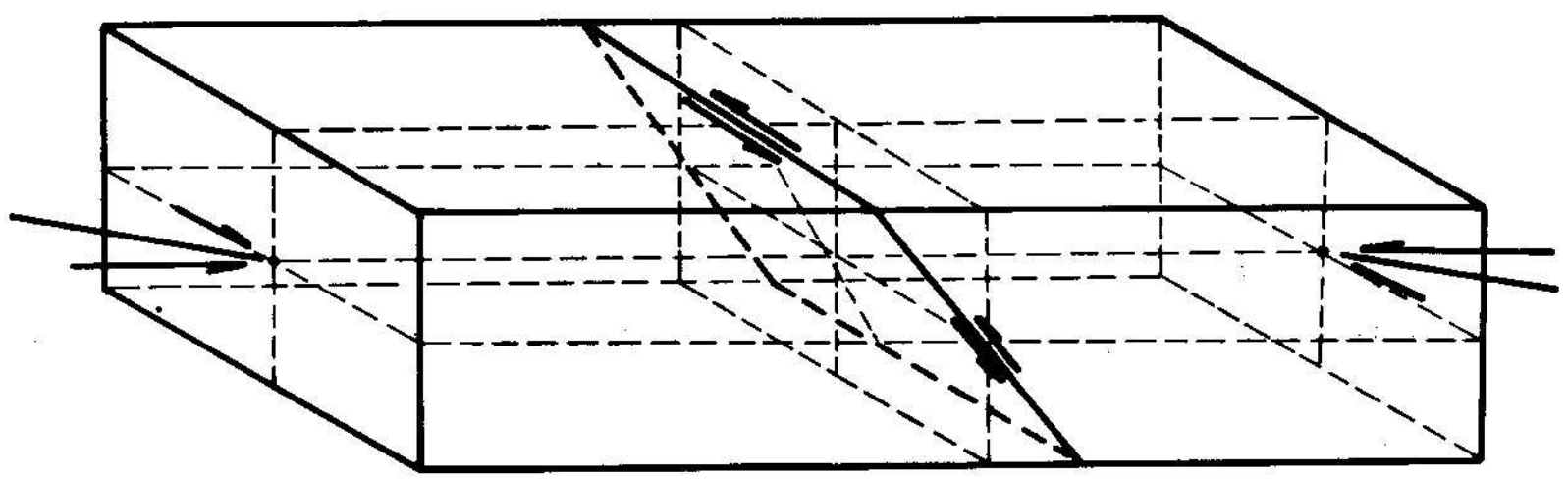

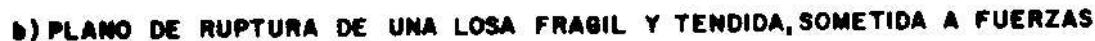
ORLICUAS Y SUS COMPONENTES HORIZONTALES. 


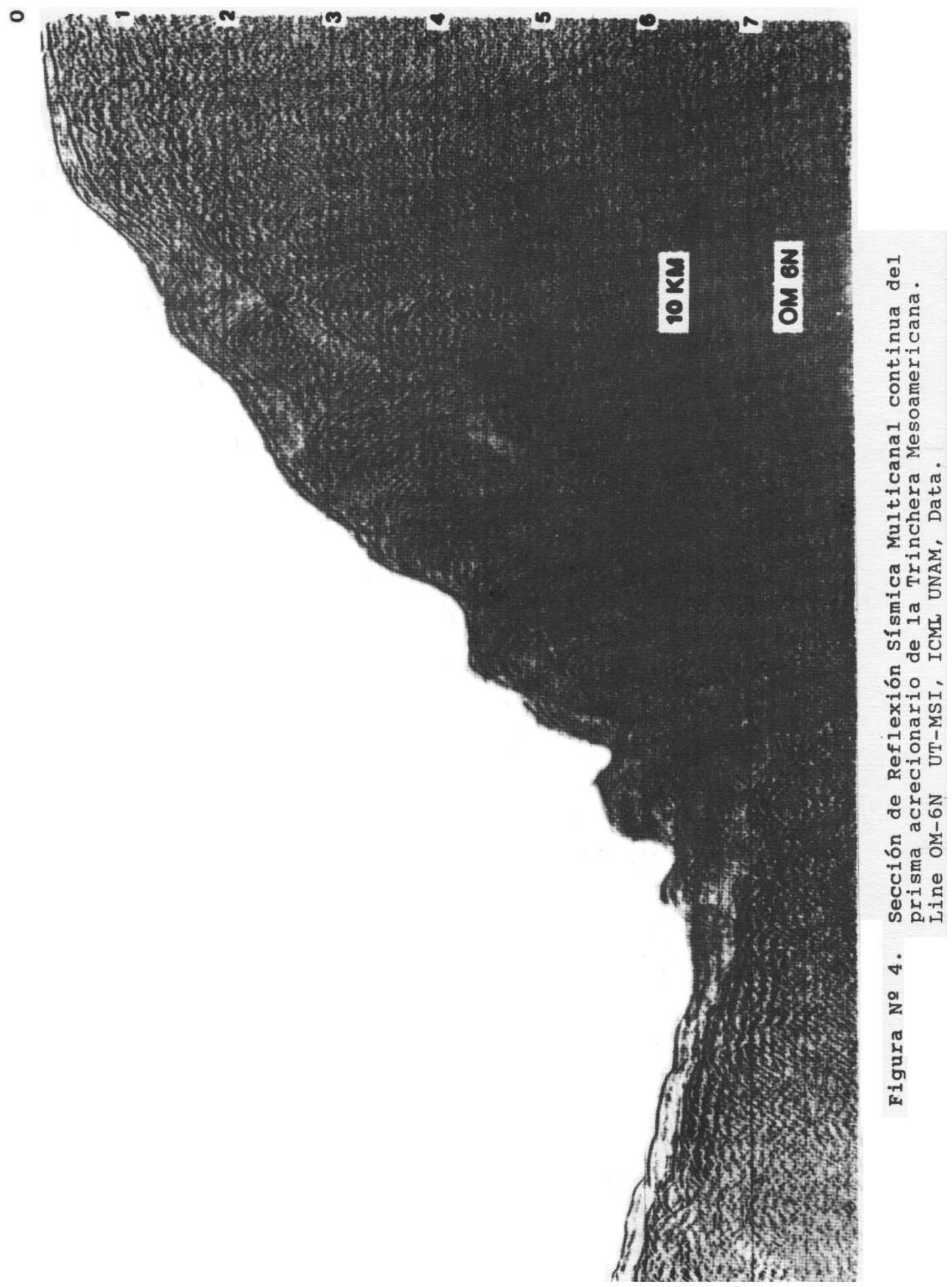




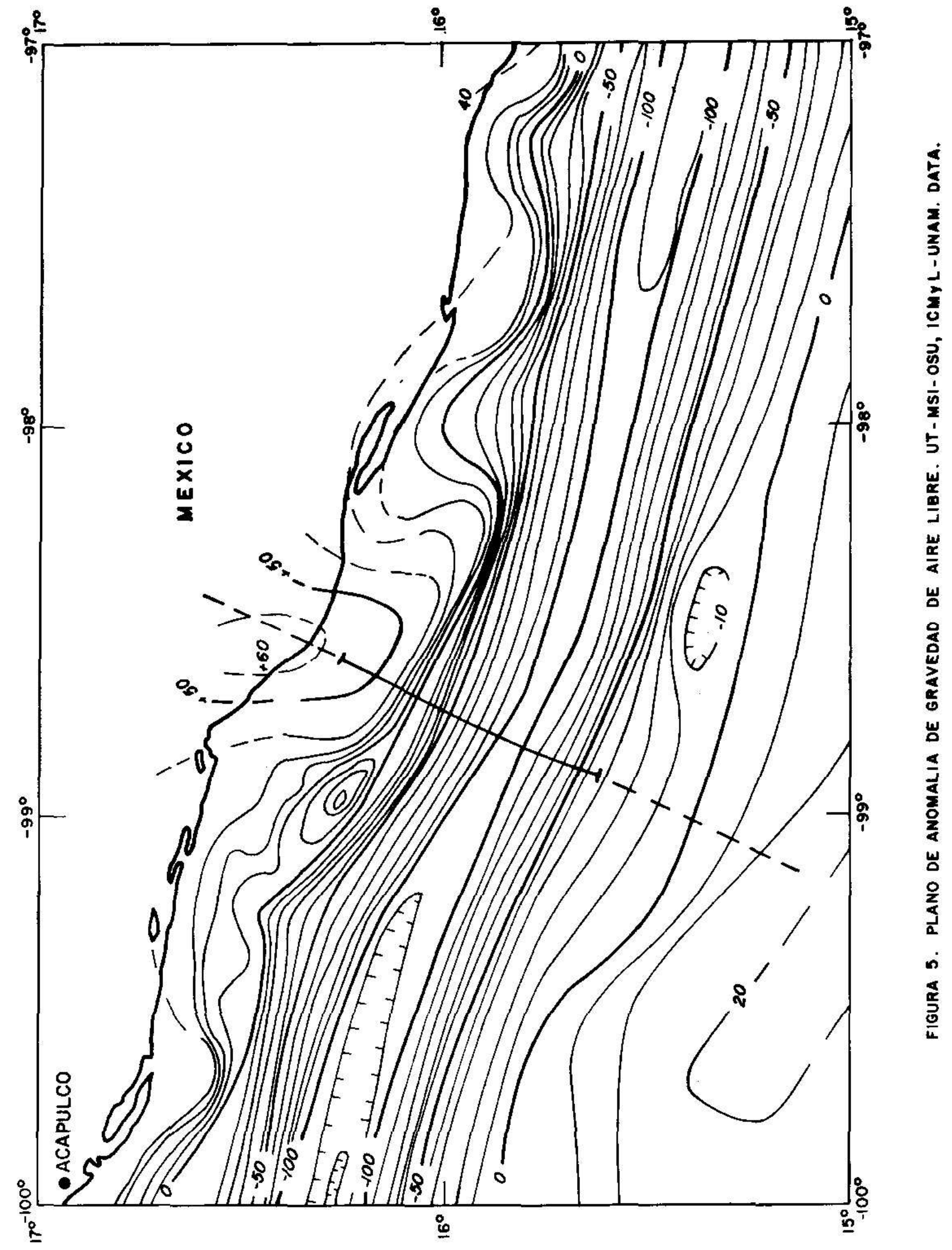



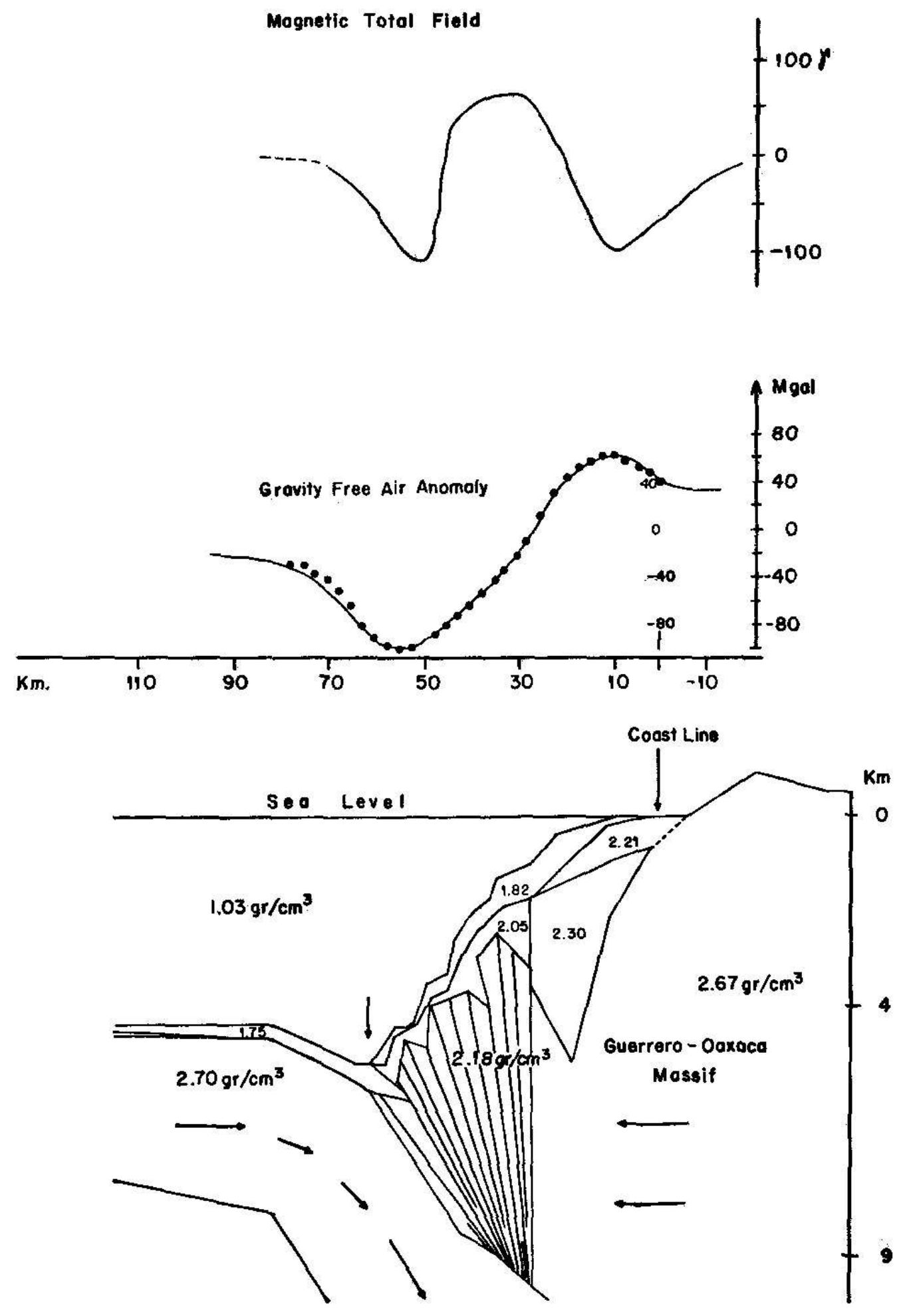

Flgura $N^{\circ} 6$ 
flexión de litosfera oceánica, antes de la subducción, genera un alto gravimétrico relativo en el lado hacia el mar, más allá de la Trinchera (Cuch y Woodcock 1981); esa misma porción, cuando avanza hacia el continente rebasando el eje de la Trinchera, genera un bajo gravimétrico relativo, exactamente por debajo del Talud y la Trinchera conllevando lo siguiente: a) Los depósitos ligeros del cuerpo Neógeno. b) No obstante que bajo esta región la corteza oceánica es un poco más gruesa, se ha alejado, debido al buzamiento. c) Ahí mismo por debajo de la misma zona el también Manto Superior, más denso también ha sido desplazado. Inicialmente la notable influencia debida a los efectos tensionales y de flexión, en la parte superior de la placa se han creado huecos, hendiduras, fosas de trinchera cual si fueran grabens; espacios que son ocupados por el agua marina y los sedimentos ligeros no compactados como los de corbetera de la placa oceánica; turbidíticos, pelágicos y hemipelágicos. Creando una masa anómala en defecto de tal manera que el bajo gravimétrico se explica.

En el año de 1948, Melnesz (en Couch y Woodcock, 1981), reportó cuatro mediciones de gravedad sobre la Trinchera a lo largo de la longitud $98^{\circ} 20^{\prime}$ Oeste, cerca de la sección estudiada. Así también reportaron, que en esta región, la Trinchera y el Talud continental están asociados con una evidente falta de compensación isostática. Después de J.C. Moore et. al., (1981) se deduce cómo es que el prisma acrecionario por su anomalía isostática negativa (De la O' Carreño, 1949), tiene una sobre compensación isostática original y que tal estructura tal como un bloque, hoy en día continúa levantándose, manteniendo o incrementando su sobre compensación. Es decir, el nuevo prisma continental para poder ascender su relieve necesita comportarse como un volumen creciente de corteza y lo logra mediante el proceso de enjarre continuo de emplastaduras múltiples, debajo de la base (under-plating) del prisma (Watkins et. al., op. cit.). Para esto es necesario incrementar o al menos mantener constante su "normal" sobre compensación isostática. Siendo así, no se esperará que el espesor subnormal del prisma y su peso ligero sean congruentes con su continuo levantamiento y creciente sobrecompensación.

Además en otro sentido, el peso ligero del prisma dado por el relieve bajo y un próximo basamento de apoyo al prisma, en plano inclinado, permiten el acrecentamiento en su base. Este mecanismo es debido al arrastre e incorporación de las esquirlas o cuñas, producto del proceso de escarapelamiento (offscrapping) de las rocas de cobertera de la placa oceánica (Watkins, et. al., op. cit.), esas napas embrionarias cuestionadas por Arif Bult (1981).

Estas hojas de roca en parte emplazadas al prisma y en parte, especialmente, asimiladas al mismo en su basamento, reciben un esfuerzo tangencial en el plano basal inclinado debido a la componente de la fuerza horizontal del choque de placas. Asimismo, de la otra componente, normal al mismo plano base del prisma, se surte el esfuerzo tanto para el enjarre 
de emplastamiento intermitente (Watkins, et. al., op. cit.) como para contribuir con el ascenso del prisma debido a su volumen creciente. La participación simultánea de estos procesos de acción inferior del prisma y levantamiento de su relieve se da con la permanente sobrecompensación isostática de la margen continental. Por lo tanto, las condiciones de isostasia sobrecompensada por anomalía isostática negativa, en la Trinchera Norteamericana; se definen como normales, puesto que muchos otros segmentos de márgenes circumpacíficas, aún maduras, acusan, típicamente, anomalías isostáticas negativas y se han reportado como sobrecompensadas. O sea, que en esta región investigada, al igual, aparentemente que muchas otras, el prisma acrecionario, nace delgado, ligero y hundido conservando estas condiciones durante su juventud y madurez, proporcionalmente con su sobrecompensación isostática.

\section{ASPECTOS CONCLUYENTES E IMPLICACIONES:}

No obstante que, como consideran otros autores, la compensación isostática se propaga más allá del área del orogénesis (Gunn, 1937) o bien, para áreas de extesión limitada (menos de 100 $\mathrm{km}$ ) predomina la compensación isobárica (Woollard y Monges, 1956); el análisis isostático cualitativo se ha realizado aquí, para el prisma acrecionario a partir de la información de las anomalías isostáticas de ésta y de otras márgenes continentales circumpacíficas, y que han solicitado modelos de cuerpos acrecionarios como éste, en una zona local con un estado regional de esfuerzos compresionales horizontales que se equilibran. Así, el prisma se ha sometido a un sistema cambiante de esfuerzos tensionales y compresionales bien definido, donde el papel de la gravedad ha prevalecido, de tal manera que la respuesta es el crecimiento de la corteza que conlleva el desarrollo de una zona de transición (transition zone) aparentemente compleja.

La primera etapa de la evolución geodinámica en la región fue el truncamiento debido a la reorganización de las placas y que afectó al macizo de Guerrero-Oaxaca como resultado de los esfuerzos de una subducción oblicua durante la época de transición Ollgo-Miocénlca. (22.5 Mo). Esta erosión tectónica regional ha tenido lugar por lo menos una vez, desde entonces, hasta 10 Ma. Sin embargo después evolucionó una acumulación secuencial continua de cuñas sedimentarias con el proceso de escarapelamiento de esquirlas de la roca de cobertera, propiciado por el fracturamiento normal que se presenta por la flexión de la placa al acercarse a la Trinchera. $Y$ así, en dirección hacia el continente exactamente, por debajo del pie del talud continental inferior, que forma la pared interna de la Trinchera; las cuñas desgajadas se deslizan unas respecto a otras sobre los mismos planos de falla que una vez fueron de fallas directas, y que ahora ahí, actúan como fallas rotacionales inversas. A causa del estado de esfuerzos compresionales también limitan su extensión inferior en la zona tanto estas fallas como las cuñas, en la zona de 
emplastamiento del prisma acrecionario. Estas cuñas ya apiladas de manera inversa se deslizan entre sí, como naipes, sobre sus planos de falla rotacional con un efecto de decollement local entre ellas. El avance de las napas inferiores impulsa. al conjunto y con ello genera las protuberancias que dan forma de "jorobas" escalonadas al talud continental. En ocasiones estas protuberancias 0 altos estructurales constituyen trás de sí, verdaderas cuencas cuaversales (Sandoval-Ochoa, 1985) que al azolvarse crean evidentes terrazas o remansos tectónicos. Las estructuras así generadas en este tipo de márgenes son las que ofrecen los mejores atractivos potenciales de recursos naturales. En un panorama general, la dinámica en las estructuras, entre ambas regiones vecinas a la Trinchera, se nota de inmediato que a lo largo de su eje el contraste tectónico es muy evidente, supuesto que, la fosa de la Trinchera misma y el receptáculo del prisma, han sido originados por los efectos extensionales de la flexión y fragilidad de la parte alta de la placa. En consecuencia, inmediatamente después, en el talud continental inferior con el receptáculo ocupado por el prisma, también está presente otro dominio de esfuerzos, ahora compresionales que son absorbidos por dicho prisma acrecionario. A lo largo del tiempo geológico, en términos relativos, el proceso de subducción ha tenido lugar con un movimiento estacionario muy rápido, y no así, ha sido el proceso de emplastamiento múltiple en la base del prisma, el cual ha sido muy lento. La conversión de efectos tensionales a compresionales sobre las mismas piezas o cuñas escarapeladas (offscrapped pieces) formadas con trozos de carpetas de pelágicos y hemipelágicos y ocasionalmente esquirlas de corteza oceánica o melanges, ahí Intercaladas; tienen como resultado un crecimiento en volumen del prisma acrecionario a expensas del material aportado en los procesos de escarapelamiento y emplastamiento múltiple sobre la placa oceánica en subducción. Estos procesos en el prisma, junto con el empuje isostático "restringido" propician en conjunto un levantamiento regional de la margen continental.

En tal régimen compresional de componentes de esfuerzos horizontales se da muy probablemente, una tendencia al equilibrio isobárico lateral del área puesto que las fuerzas horizontales se compensan unas a otras actuando en la sección transversal normal al empuje de placas de más de $60 \mathrm{~km}$ de grosor, aunque a lo largo del arco haya la tendencia isostática.

Así entonces, queda definido un sistema de convergencia, en equilibrio dinámico, cuyos efectos compresionales pervasivos en las placas se anulan mutuamente. En el otro sentido las fuerzas verticales resultantes, actuando en contra de la gravedad permiten la ascensión isostática del prisma acrecionario; y el levantamiento es auspiciado por los procesos descritos para el incremento de volumen de la nueva corteza dentro y encima de la coyuntura de acción entre placas.

Una vez definido el límite del continente sobre la sección analizada, se extrapoló éste sobre las otras secciones paralelas determinando así, 
sobre el plano (Figura No. 1), la traza del límite océano-continente.

En términos globales y sin proclividad hacia alguna de las teorías de la isostasia en particular, el mecanismo tendiente a la compensación isostática en la margen investigada es congruente con las anomalías de gravedad discutidas. Asimismo, de acuerdo con el modelo aquí propuesto, las anomalías de gravedad responden congruentemente al hecho de que; la variación vertical en el espesor del prisma, su dinámica, los bloques receptáculo y la variación lateral de la densidad, dominan al conjunto de la corteza transicional océano-continente. En ese marco geodinámico el mecanismo resultante del prisma responde a una sobrecompensación isostática permitiendo así que la recién nacida-neógena-corteza acrecionaria tienda a madurar con el desarrollo del acrecentamiento continental con nuevos terrenos.

\section{BIBLIOGRAFIA}

AGUAYO, C.E.J., MARIN C.S., 1987. Origen y Evolución los Rasgos Morfotectónicos. Post Cretácicos de México, Bol. Soc. Geol. Mex. V.48, 2: $15-40$.

ARIF BUTT, 1981. Sindepositional tectonics along the Middle America Trench with special reference to foraminiferal Bathymetry: Deep Sea Drilling Project Leg 66, Offshore Mexico-Geological Processes along an Active Margin. Initial Reports of JOIDES drilling DSDPGG, 29: 671-680.
CARR, M.J., R.E. STOIBER and C.L. DRAKE., 1974. The Segmented Nature of Some Continental Margins. In Burk, C.A. and C.L. Drake (Eds.) The Geology of Continental Margins. Springer Verlag N.Y., Berlin. 105-114. COUCH, R. and S. WOODCOCK, 1981. Gravity and Structure of the Continental Margin of Southwestern Mexico and Northwestern Guatemala. Jour. of Geophys. Res., 86 , B3: 1829-1840.

DE CSERNA Z., 1976. México-Geotectonics and Mineral Deposits. Reprint from New Mex, Geol. Soc., Spe. Publ. 6 (Kelly Vol.): 18-25

DE LA O. CARREÑO, A., 1949. Cartas de anomalías de la Gravedad en la República Mexicana.

GARLAND, G.D., 1970. The Earth's Shape and Gravity, The Commonwealth and Intl. Library, Pergamon Press, N.Y. 183 pp.

GUNN, ROSS. A., 1937. A quantitalive study of Mountain Building on an unsymmetrical Earth, Jour. of the Franklin Institute, V. 224, No. 1.

HANDSCHUMACHER, DW., 1976. Post-Eocene plate tectonic in the eastern Pacific. In Sutton, G.H., Manghnani, M.H., and Moberly, R. (Eds.), The Geophysics of the Pacific Ocean Basin and its Margins: Am. Geophys. Union Monogr., 19: 177-202.

KARIG, D.E., 1974. Tectonic Erosion at Trenches. Earth and Planet Sci. Letters, N. Holland Publ. Comp. Anstm. Print Netherlands, 21: 209-212. KARIG, D.E., R.K. CALDWELL, G. F. MOORE et. al., 1978. Late Cenozoic Subduction and Continental Margin Truncation along the northern 
Middle America Trench. Geol. Soc. Am. Bull., 89:265-276.

MALFAIT, B.T. and M.G. DINKELMAN, 1972. Circum Caribbean, tectonic and igneous activity and the evaluation of the Caribbean plate. Geol. Soc. Am. Bull. 83: 205-272.

MOORE, J.C., J.S. WATKINS and T.H. SHIPLEY. 1981. Summary of accretionary processes, Deep Sea Drilling Project Leg 66: Offscrapping, Underplating, and Deformation of the Slope Apron.

Initial Reports of JOIDES drinlling DSDP 66, 42: 825-836.

NEIL LUNDBERG and J.C. MOORE, 1981. Structural Features of the Middle America Trench Slope off Southern Mexico, Deep Sea Drilling Project Leg 66. Initial Reports of JOIDES drilling DSDP 66, 40: 793-814. SANDOVAL-OCHOA J.H., 1985. The Tecpan Regional Fault: Evidence for Major NE Lineaments. Geof. Int. 24, 1: 193-202. SEELY, D.R., P.R. VAIL and G.G. WALTON., 1974. Trench Slope Model. In Burk, C.A. and C.L. Drake (Eds.), The Geology of Continental Margins. Springer Verlag, N.Y., Berlin. 249-260. SHIPLEY, IH., K.J. MCMILLEN, J.S. WATKINS, J.C. MOORE, J.H.

SANDOVAL-OCHOA and J.L. WORZEL, 1980. Continental Margin and Lower Slope Structures of the Middle America Trench near Acapulco (Mexico). Marine Geol. 35: 65-82.

SHIPLEY T.H., 1981. Seismic Facies and Structural Framework of the Southern Mexico
Continental Margin. Initial Reports of the JOIDES Drilling Deep Sea Drilling Project, 66, 39: 775-790.

STOIBER, R.E., and M.J. CARR., 1973. Quaternary Volcanic Segmentation of Central America. Bull. Vulcanologique. 37, No. 3: 304-325.

TALWANI, M.J.L. WORZEL and M. LANDISMAN, 1959. Rapid Gravity Computations for two dimensional bodies with applications to the Mendocino Submarine Fracture Zone. Jour. Geophys. Res., 64, 10: 49-59.

TRUCHAN, MARCK and ROGER L. LARSON., 1974. Tectonic Lineaments on the Cocos Plate. Earth and Planetary Science Letters, N-Holland Publ. Comp., 17: 426-432.

VENING MEINESZ, F.A., 1948. Gravity Expeditions at sea 1923 1938. Report, Neth. Geod. Comm; Delftche Vitgevers Mij, Delf, The Netherlands.

VERMA, S.P., 1985. Mexican Volcanic Belt (Preface), In Verma, S.P. (Guest Edr.) Mexican Volcanic Belt, part 1. Geofísica Internacional, 24, 1: 7-18.

WATKINS, J.S., K.J. McMILLEN, S.B. BACHMAN, et.. al., 1981. Tectonic Synthesis, Leg 66: Transect and Vicinity, Initial Reports of JOIDES drilling DSDPGG, 43: 837-849.

WOOLLARD, G.P. and J. MONGES CALDERA, 1956. Gravedad, Geología Regional y Estructura Cortical en México. Anales del Instituto de Geofísica, UNAM. 2, 60-112. 